\title{
Comparison of Two Different Synthesis Methods of Single Crystals of Superconducting Uranium Ditelluride
}

\author{
Sheng Ran ${ }^{1,2,3}$, I-Lin Liu ${ }^{1,2}$, Shanta R. Saha ${ }^{1,2}$, Prathum Saraf ${ }^{1}$, Johnpierre Paglione ${ }^{1,2}$, Nicholas P. Butch ${ }^{1,2}$ \\ ${ }^{1}$ Maryland Quantum Materials Center, Department of Physics, University of Maryland ${ }^{2}$ National Institute of Standards and Technology ${ }^{3}$ Department of \\ Physics, Washington University in St. Louis
}

\section{Corresponding Author}

Nicholas P. Butch

nbutch@umd.edu

\section{Citation}

Ran, S., Liu, I.L., Saha, S.R., Saraf, P., Paglione, J., Butch, N.P. Comparison of Two Different Synthesis Methods of Single Crystals of Superconducting Uranium Ditelluride. J. Vis. Exp. (173), e62563, doi:10.3791/62563 (2021).

\section{Date Published}

July 8,2021

\section{DOI}

$10.3791 / 62563$

URL

jove.com/video/62563

\section{Abstract}

Single crystal specimens of the actinide compound uranium ditelluride, UTe2, are of great importance to the study and characterization of its dramatic unconventional superconductivity, believed to entail spin-triplet electron pairing. A variety in the superconducting properties of $\mathrm{UTe}_{2}$ reported in the literature indicates that discrepancies between synthesis methods yield crystals with different superconducting properties, including the absence of superconductivity entirely. This protocol describes a process to synthesize crystals that exhibit superconductivity via chemical vapor transport, which has consistently exhibited a superconducting critical temperature of $1.6 \mathrm{~K}$ and a double transition indicative of a multi-component order parameter. This is compared to a second protocol that is used to synthesize crystals via the molten metal flux growth technique, which produces samples that are not bulk superconductors. Differences in the crystal properties are revealed through a comparison of structural, chemical, and electronic property measurements, showing that the most dramatic disparity occurs in the low-temperature electrical resistance of the samples.

\section{Introduction}

At temperatures typically much lower than room temperature, many materials exhibit superconductivity-the fascinating macroscopic quantum state in which the electrical resistance becomes absolutely zero and electrical current can flow without dissipation. In the typical superconducting phase, instead of acting as separate entities, the constituent electrons form Cooper pairs, which are commonly made up of two electrons with opposite spins, in a spin singlet configuration. However, in very rare cases, the Cooper pairs can instead be made up of two electrons with parallel spins, in a spin triplet configuration. Among the few thousand superconductors discovered so far, there are only a few superconductors that have been identified as spin triplet candidates. This rare quantum phenomenon has attracted a lot of research interest because spin triplet superconductors are proposed to be one potential building 
block for quantum computers ${ }^{1,2}$, the next generation of computation technology.

Recently, Ran and coworkers reported that $\mathrm{UTe}_{2}$ is a candidate spin triplet superconductor ${ }^{3}$. This superconductor has many exotic properties indicative of a spin triplet configuration: an extreme, disproportionately large, critical magnetic field required to suppress the superconductivity, a temperature-independent NMR Knight shift ${ }^{3}$, a spontaneous magnetic moment indicated by the optical Kerr effect ${ }^{4}$, and a chiral electronic surface state indicated by scanning tunneling spectroscopy ${ }^{5}$. Moreover, additional superconducting phases are actually induced in high magnetic field ${ }^{6}$, an example of the unusual phenomenon of reentrant superconductivity.

Although these new results are robust, the superconducting properties of UTe2 depend on the synthesis process used by different groups ${ }^{7,8,9}$. Crystals of UTe2 synthesized using the chemical vapor transport method superconduct below a critical temperature of $1.6 \mathrm{~K}$. In contrast, those grown using the molten flux method have a greatly suppressed superconducting critical temperature or do not superconduct at all. In anticipation of applications such as quantum computing, reliably obtaining crystals that superconduct is highly desirable. Moreover, investigating why nominally similar crystals do not superconduct is also very helpful for understanding the fundamental superconducting pairing mechanism in UTe2, which, while novel and a topic of intense research, must differ significantly from that of conventional superconductors. For these reasons, the two different synthesis methods are complementary and useful to compare. In this paper, two different methods for synthesis of $\mathrm{UTe}_{2}$ are demonstrated and properties of the single crystals from the two methods are compared.

\section{Protocol}

\section{Removing uranium oxide from the uranium metal}

1. In a fume hood, prepare three beakers that contain $1 \mathrm{~mL}$ of nitric acid, $5 \mathrm{~mL}$ of distilled water, and $5 \mathrm{~mL}$ of acetone, respectively.

2. Using a saw or cutters, cut a piece of uranium metal to the desired mass.

3. Using tweezers, place the uranium into the beaker with nitric acid. Wait for approximately $10 \mathrm{~s}$ for the acid to dissolve the black oxidized surface, such that the uranium appears shiny and metallic.

4. Take out the piece of uranium from the acid, rinse it in distilled water for $5 \mathrm{~s}$, and then remove the piece of uranium.

5. Put the uranium into the beaker containing acetone for 5 $s$ and remove it.

6. Determine the mass of the uranium. The uranium is ready for synthesis.

\section{Chemical vapor transport}

1. Weigh out an appropriate amount of elemental tellurium, depending on the amount of uranium previously cleaned, following an atomic ratio of uranium to tellurium of $2: 3$.

2. Weigh out an appropriate amount of iodine, determined by the desired density of $1 \mathrm{mg} / \mathrm{cm}^{3}$ in the volume of the quartz tube to be used during synthesis. Choose a length of the tube to span the furnace, with each end sitting in one of the temperature zones. Ensure that the diameter fits well in the furnace. 
NOTE: Samples previously reported $^{3}$ were made in a quartz tube that is $11 \mathrm{~cm}$ long, with a $14 \mathrm{~mm}$ inner diameter, so that the total mass of iodine is about $17 \mathrm{mg}$.

3. Close one end of a fused quartz tube using a torch. A hydrogen/oxygen torch works well. Use any torch that gets hot enough to soften fused quartz. Allow the tube to cool.

4. Put all the materials into the quartz tube. Evacuate the tube using a dry vacuum pump and seal the tube with the torch.

5. Insert the tube into a 2-zone horizontal tube furnace. When doing this, make sure to slide all the raw materials to one side of the tube, which will be the hot side.

6. Over $12 \mathrm{~h}$, heat at constant rates the hot side to $1060^{\circ} \mathrm{C}$, and the other side to $1000{ }^{\circ} \mathrm{C}$. Hold the temperatures for 1 week, and then turn off the furnace to allow it to cool slowly to room temperature.

\section{Molten metal flux growth}

1. Weigh out uranium and tellurium according to the atomic ratio of 1:3.

2. Put all the materials in a $2 \mathrm{~mL}$ alumina crucible. On top of this crucible, place another $2 \mathrm{~mL}$ crucible, filled with quartz wool, facing downward.

3. Close one end of a fused quartz tube using a torch. A hydrogen/oxygen torch works well. Use any torch that gets hot enough to soften the fused quartz. Allow the tube to cool.

4. Place the two crucibles into a quartz tube having a 14 $\mathrm{mm}$ inner diameter. Use a dry vacuum pump to evacuate the tube and then seal the tube with the torch.
5. Put the quartz tube into a $50 \mathrm{~mL}$ alumina crucible to be used as an exterior container for stability. Place these in a box furnace.

6. Over $12 \mathrm{~h}$, heat the furnace at a constant rate to $1180^{\circ} \mathrm{C}$. Hold the temperature for $5 \mathrm{~h}$. Cool the furnace at constant rate to $975^{\circ} \mathrm{C}$ for over $100 \mathrm{~h}$.

7. Prepare a centrifuge with a swing out rotor and metal buckets. At $975^{\circ} \mathrm{C}$, take out the tube using furnace tongs, carefully invert it, and then put it into the centrifuge. Spin at $2500 \times g$ (4000 rpm for these sealed quartz tube assemblies) for $10-20 \mathrm{~s}$, forcing the extra liquid tellurium to separate from the UTe2 crystals and get caught in the quartz wool.

8. Allow the tube to cool to room temperature.

\section{Opening the tubes and harvesting the crystals}

1. Place the quartz tube into a sealed plastic bag and place it on a hard surface, such as a laboratory benchtop or a fume hood.

2. Using a small hammer or any other blunt object, carefully crack and break the quartz tube, preferably at the end away from the crystals.

3. Open the plastic bag and pick out the UTe2 crystals. Perform this process within an hour or so, as $\mathrm{UTe}_{2}$ is air-sensitive and noticeably degrades over the course of several hours.

4. Rinse the crystals with $2 \mathrm{~mL}$ of ethanol to remove iodine.

5. Store the UTe2 crystals under an inert atmosphere, such as inside a nitrogen glove box. 


\section{Representative Results}

Both growth techniques yield crystals of $\mathrm{UTe}_{2}$ having dimensions on the millimeter length scale. Crystals are shiny, with a metallic luster. The crystal morphology is variable, and intergrowths can occur. Generally, chemical vapor transport and flux grown crystals look similar and are not easily distinguishable by visual inspection, as is evident in Figure 1.

To confirm the crystal structure, powder x-ray diffraction measurements are typically performed on crushed single crystals of both CVT grown and flux grown $\mathrm{UTe}_{2}$ single crystals at room temperature. Single crystals from both growth techniques have the same crystal structure and are single phase, with no sign of impurity phases. Figure 2 shows the collected $x$-ray diffraction data and a refinement to a bodycentered orthorhombic crystal structure with the space group Immm ${ }^{10}$.

The temperature dependence of the electrical resistance is a typical way to characterize metallic materials. Figure 3 compares the temperature dependence of the electric resistance, normalized to the room temperature value, for samples of UTe2 synthesized using chemical vapor transport and flux methods. These data were collected in a commercial refrigerator system using a standard 4-lead configuration. Above $50 \mathrm{~K}$, both samples show a slight increase of the electrical resistance upon cooling, which is atypical of metals. This behavior is consistent with that caused by scattering of conduction electrons off the uranium atomic magnetic moments, known as the single ion Kondo effect. A broad maximum is also seen in both samples, followed by a drop in resistance due to the onset of Kondo coherence.

A distinct difference between the samples is that the value of the residual resistance, or value of the resistance in the zero-temperature limit, is dramatically larger in the sample synthesized by the flux method. The residual resistance ratio $\mathrm{RRR}$, or the ratio between the resistance value at room temperature and the residual resistance, is approximately 2 for the flux grown sample, which is about 15 times smaller than the RRR value of the chemical vapor transport sample. The greatly reduced RRR of the flux grown sample indicates that there are more crystallographic impurities or defects in the flux grown sample, which are responsible for stronger scattering of the conduction electrons, and hence the higher residual resistance. These values are consistent with previous reports ${ }^{7}$.

A more dramatic difference is that the flux grown samples do not superconduct. In general, the presence of impurities and defects is detrimental to superconductivity because increased scattering weakens the electron pairing interaction that underlies superconductivity. The effects of disorder may be even more pronounced in $\mathrm{UTe}_{2}$, in which superconductivity is believed to be of the unusual spin triplet variety that is generally more sensitive to pair breaking $11,12,13,14,15,16,17,18,19$. The effects of disorder and chemistry on superconductivity in UTe2 are still in the early days and are currently an active field of study.

The DC magnetic susceptibility, or magnetization normalized to the applied field, of both flux grown and CVT grown $\mathrm{UTe}_{2}$ look very similar. As shown in Figure 4, in which the data were collected at 1000 Oe in a commercial SQUID magnetometer, the high-temperature magnetic susceptibility shows a paramagnetic response when the magnetic field is applied along the crystallographic a-axis of the samples. At low temperatures, the magnetic susceptibility increases sharply and then shows a slight slope change at $\sim 10 \mathrm{~K}$, likely due to the Kondo coherence. The difference between the 


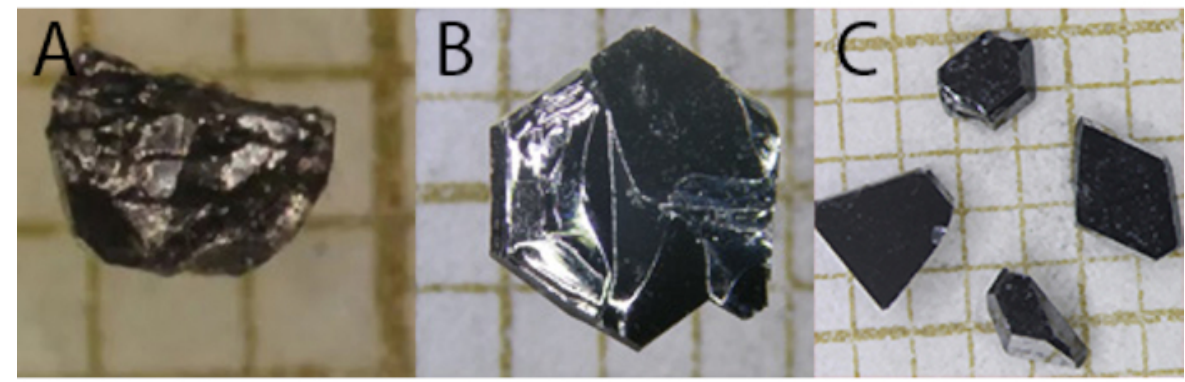

Figure 1: Photographs of single crystals of UTe2. (A) flux grown and (B-C) CVT grown. The grids are $1 \mathrm{~mm}$. Please click here to view a larger version of this figure.

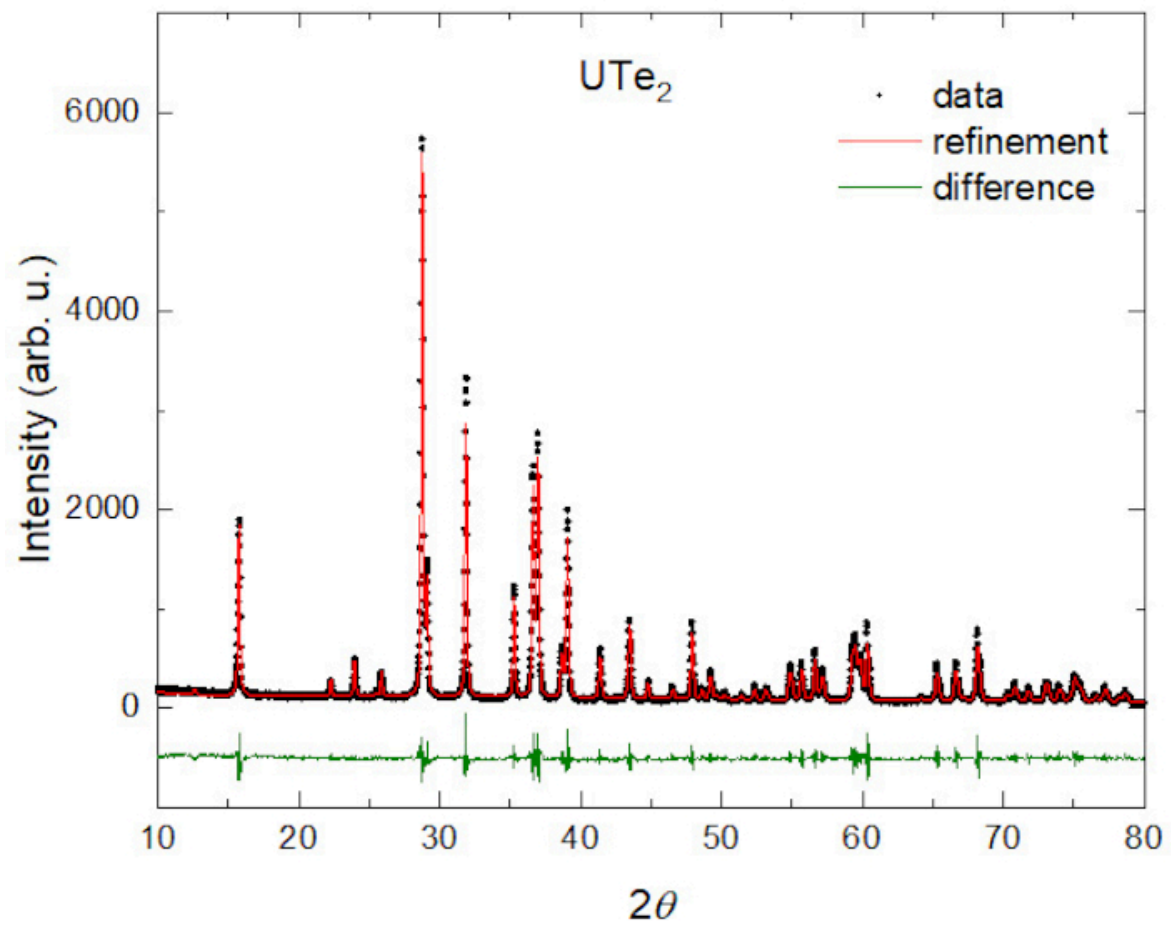

Figure 2: Powder Xray diffraction data of CVT grown UTe2. The data shows the good quality of the sample with no visible peaks from impurities. Please click here to view a larger version of this figure. 


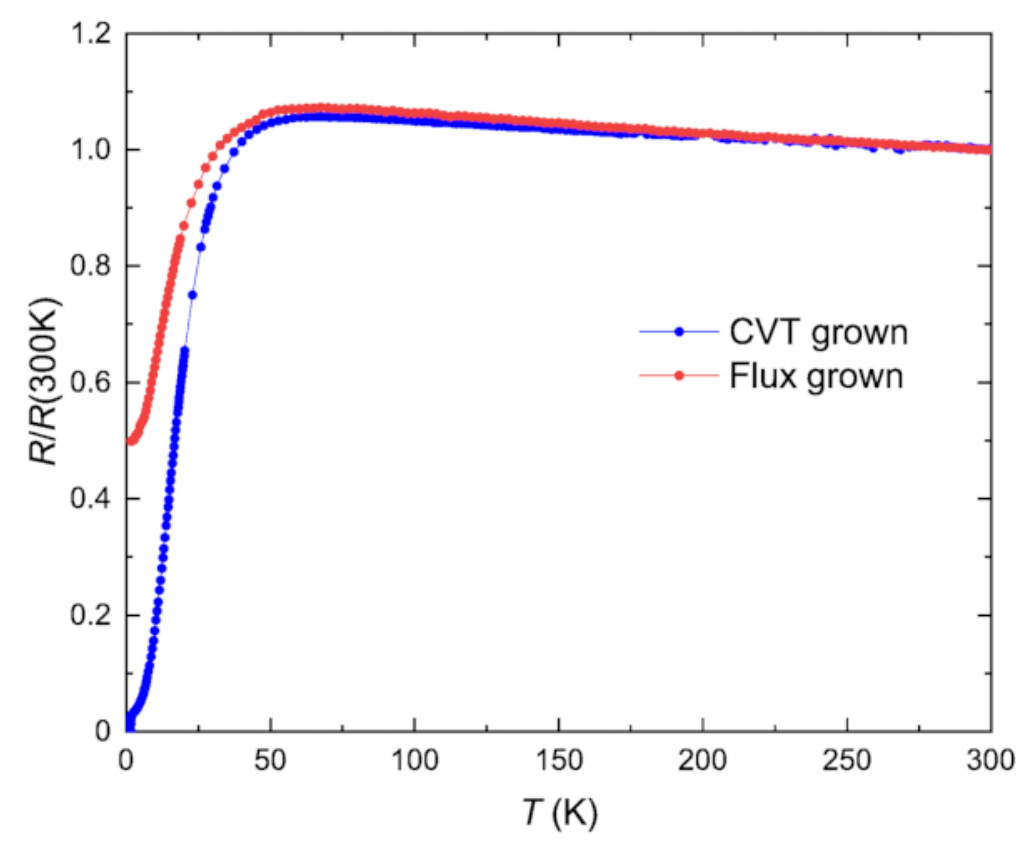

Figure 3: Normalized electrical resistance data as a function of temperature for both CVT grown and flux grown

UTe2. The flux grown sample has a substantially larger residual resistance, which is a signature of increased crystallographic disorder. Please click here to view a larger version of this figure. 


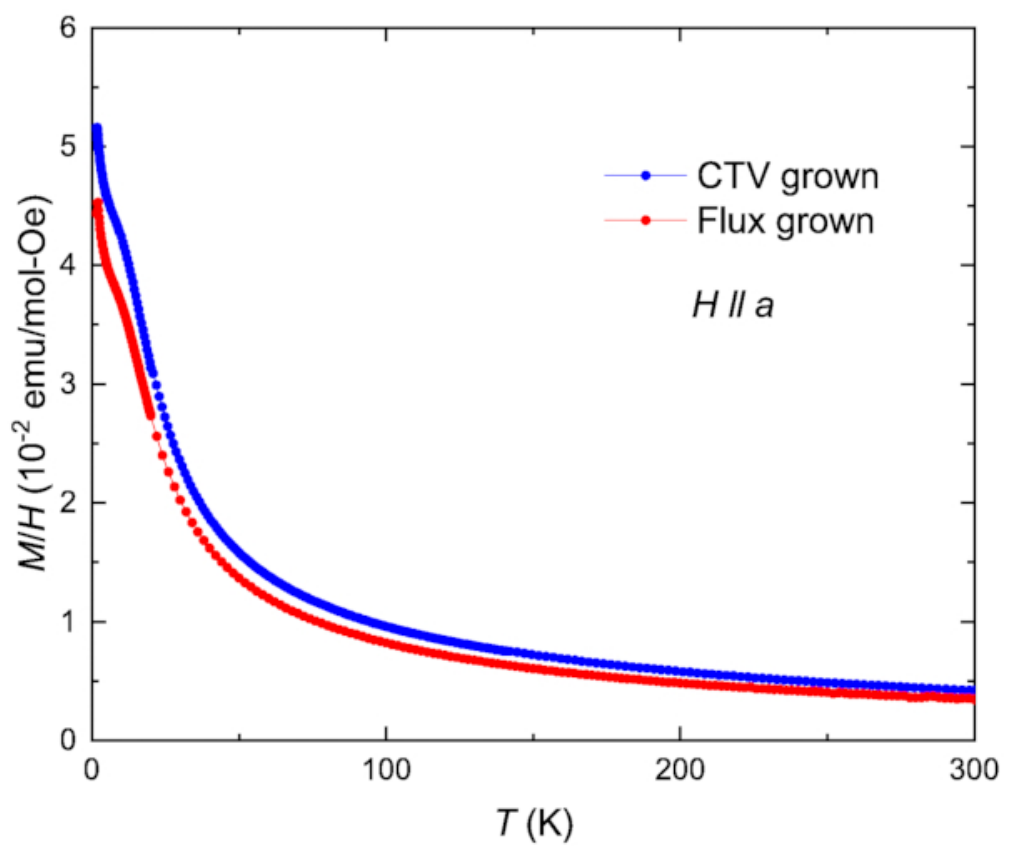

Figure 4: Magnetic susceptibility, or magnetization normalized to applied magnetic field, as a function of temperature for both CVT grown and flux grown UTe2. The samples show similar behavior, including a characteristic kink at approximately $10 \mathrm{~K}$. A magnetic field $\mathrm{H}=1000$ Oe is applied parallel to the crystallographic a-axis. Please click here to view a larger version of this figure.

\section{Discussion}

To perform chemical vapor transport, it is simplest to utilize a two-zone horizontal furnace, which can generate a temperature gradient by setting the two zones at different temperatures. Successful use of a one-zone furnace to grow superconducting samples has not yet been demonstrated. Starting materials are sealed with a hydrogen-oxygen torch in a fused quartz tube, which must be purged of air. The purging and sealing can be accomplished by connecting the tube to a manifold attached to a dry pump and an argon gas cylinder. Once prepared, this tube is placed in the furnace such that two ends of the tube span the two temperature zones. In the case of $\mathrm{UTe}_{2}$, the end of the tube containing the starting materials is placed at the hot end. The elemental uranium and tellurium react with iodine, travel down the tube as a vapor, and eventually solidify at the cold end the quartz tube in the form of single crystals. Generally, the growth of large crystals is material-dependent and can take several weeks. For $\mathrm{UTe}_{2}, 7$ days is enough to grow crystals with $\mathrm{mm}$ dimensions. Following the growth, the tube is removed from the furnace and opened to harvest the crystals.

The molten metal self-flux method requires a simple resistive box furnace with one temperature zone. Uranium dissolves in molten tellurium, and the solubility of $\mathrm{UTe}_{2}$ is dependent on temperature. Starting materials, elemental uranium, and tellurium, are placed in an alumina crucible. On top of this crucible, a second crucible is placed upside down, filled with quartz wool. The two crucibles are sealed in a quartz tube, 
which is put in a box furnace. This time, instead of generating a fixed temperature gradient over a distance, the temperature is varied as a function of time, as the furnace is slowly cooled at a fixed rate. At the highest temperature, all the uranium will be dissolved in liquid tellurium, which has a much lower melting temperature than uranium. As the furnace cools, the solubility of $\mathrm{UTe}_{2}$ decreases and $\mathrm{UTe}_{2}$ single crystals precipitate and get larger. At a temperature that is low enough to have generated sufficiently large UTe2 single crystals, but still high enough for the tellurium to remain liquid, the quartz tube is removed from the hot furnace, placed it into a centrifuge and spun, which separates the solid UTe2 from the liquid tellurium before it freezes. After that, the tube is allowed to cool to room temperature, before it is broken to collect the crystals.

Working with depleted uranium is a heavily regulated activity that requires awareness of and compliance with applicable laws. Follow all local applicable hazardous and radioactive materials safety rules, and secure necessary permission to perform this work. These rules vary by jurisdiction and institution and cannot be addressed here. However, some general principles apply that can help with the planning of research. Researchers should be trained to work with radioactive and hazardous materials. Wear necessary personal protective equipment, including gloves. Work methodically and take care to avoid the spread of radioactive material. Discard waste in labeled and approved containers.

\section{Disclosures}

The authors declare no competing interests.
This research was supported by the National Institute of Standards and Technology. Portions of the synthesis were supported by Gordon and Betty Moore Foundation's EPiQS Initiative through Grant No. GBMF9071. Portions of the characterization were supported by the US Department of Energy (DOE) award DE-SC0019154. Identification of certain commercial products and company names is not intended to imply recommendation or endorsement by the National Institute of Standards and Technology, nor is it intended to imply that the products or names identified are necessarily the best available for the purpose.

\section{References}

1. Sau, J. D., Tewari, S. Topologically protected surface majorana arcs and bulk weyl fermions in ferromagnetic superconductors. Physical Review B. 86 (10), 104509 (2012).

2. Fu, L., Kane, C. L. Superconducting proximity effect and majorana fermions at the surface of a topological insulator. Physical Review Letters. 100 (9), 096407 (2008).

3. Ran, S. et al. Nearly ferromagnetic spin-triplet superconductivity. Science. 365 (6454), 684-687 (2019).

4. Hayes, I. M. et al. Weyl Superconductivity in UTe2. arXiv. 2002.02539 (2020).

5. Jiao, L. et al. Chiral superconductivity in heavy-fermion metal UTe2. Nature. 579, 523 (2020).

6. Ran, S. et al. Extreme magnetic field-boosted superconductivity. Nature Physics. 15, 1250-1254 (2019).

\section{Acknowledgments}


7. Aoki, D. et al. Unconventional superconductivity in heavy fermion UTe2. Journal of the Physical Society of Japan. 88, 043702 (2019).

8. Cairnsm, L. P., Stevensm, C. R., O'Neill, C. D., Huxley, A. Composition dependence of the superconducting properties of UTe2. Journal of Physics. Condensed Matter: An Institute of Physics Journal. 32 (41), 415602 (2020).

9. Thomas, S. M. et al. Evidence for a pressure-induced antiferromagnetic quantum critical point in mixed valence UTe2. Science Advances. 6 (42), eabc8709 (2020).

10. Hutanu, V. et al. Low-temperature crystal structure of the unconventional spin-triplet superconductor $\mathrm{UTe}_{2}$ from single-crystal neutron diffraction. Acta Crystallographica Section B, Structural Science, Crystal Engineering and Materials. 76 (Pt 1), 137-143 (2020).

11. Sundar, S. et al. Coexistence of ferromagnetic fluctuations and superconductivity in the actinide superconductor UTe2," Physical Review B. 100, 140502(R) (2019).

12. Metz, T. et al. Point-node gap structure of the spintriplet superconductor UTe2. Physical Review B. 100, 220504(R) (2019).

13. Knebel, G. et al. Field-reentrant superconductivity close to a metamagnetic transition in the heavy-fermion superconductor UTe2. Journal of the Physical Society of Japan. 88, 063707 (2019).

14. Braithwaite, D. et al. Multiple superconducting phases in a nearly ferromagnetic system. Communications Physics. 2, 147 (2019).
15. Ran, S. et al. Enhancement and reentrance of spin triplet superconductivity in UTe2 under pressure. Physical Review B. 101, 140503(R) (2020).

16. Nakamine, G. et al. Superconducting properties of heavy fermion UTe2 revealed by ${ }^{125}$ Te-nuclear magnetic resonance. Journal of the Physical Society of Japan. 88, 113703 (2020).

17. Miao, L. et al. Low energy band structure and symmetries of UTe2 from angle resolved photoemission spectroscopy. Physical Review Letters. 124, 076401 (2020).

18. Lin, W.-C. et al. Tuning magnetic confinement of spintriplet superconductivity. npj Quantum Materials. 5, 68 (2020).

19. Bae, S. et al. Anomalous normal fluid response in a chiral superconductor. arXiv. 1909.09032 (2019). 